\title{
ANÁLISIS DE LA VULNERABILIDAD BIOFÍSICA A LOS RIESGOS POR INUNDACIÓN EN LA ZONA METROPOLITANA DE TOLUCA, MÉXICO
}

\author{
Jesús Baruch Mendoza Mejía ${ }^{1}$ \\ María Estela Orozco Hernández ${ }^{2}$
}

Recibido el 16 de septiembre de 2012 y aprobado el 5 de julio de 2013

\section{RESUMEN}

Se realizó el análisis multicriterio de los factores físico-naturales de la Zona Metropolitana de Toluca (ZMT), con la finalidad de definir el índice biofísico de exposición a los riesgos por inundación. Para la elaboración del trabajo se utilizó información institucional e información de campo, la cual fue procesada a través de los métodos estadísticos y multicriterio con el apoyo de los Sistemas de Información Geográfica y de percepción remota. Los resultados obtenidos mostraron que el índice de riesgo alto, con un valor de $52,41 \%$ comprende los municipios de Lerma, Toluca, San Mateo Atenco y Metepec y cubre una superficie de 1032,73 km2; el índice bajo cubre el $13,71 \%$ del territorio y una superficie $13,71 \mathrm{~km}^{2}$; por último el riesgo biofísico medio abarca $687,20 \mathrm{~km}^{2}$ o $33,88 \%$ del territorio metropolitano. Se pretende que los resultados de la investigación contribuyan en la toma de decisiones en los campos de la protección civil, el desarrollo regional y la seguridad pública, así como la incorporación en los planes de desarrollo urbano y ordenamiento territorial con una visión de riesgo; y sea de utilidad en los campos de la protección civil, el desarrollo metropolitano, la seguridad pública y el ordenamiento territorial.

\section{PALABRAS CLAVE}

Vulnerabilidad, amenaza, riesgo, inundación, multicriterio.

\section{BIOPHYSICS VULNERABILITY ANALYSIS TO FLOODING RISK IN THE METROPOLITAN AREA OF TOLUCA, MEXICO}

\section{ABSTRACT}

A multicriteria analysis of natural physical factors of the Toluca Metropolitan Area (ZMT) was performed in order to define the biophysical index of exposure to flood risk. Institutional information and field information was used for carrying out the work and it was processed through statistical and multi-criteria methods with the support of GIS and remote sensing. The results showed that the rate of high risk, with a $52.41 \%$ value includes the municipalities of Lerma, Toluca, San Mateo Atenco and Metepec, and covers an area of $1032.73 \mathrm{~km} 2$. The low rate covers $13.71 \%$ of the territory and an area of $13.71 \mathrm{~km} 2$, and finally the average biophysical risk covers $687.20 \mathrm{~km} 2$ or $33.88 \%$ of the metropolitan area. It is intended that the research results contribute to decision making in the fields of civil protection, regional development and public safety, as well as incorporation into urban development plans and land use planning 
with a view of risk, and be useful in the fields of civil protection, metropolitan development, public safety and land use planning.

\section{KEYWORDS}

Vulnerability, threat, risk, flood, multicriteria.

\section{INTRODUCCIÓN}

La probabilidad de ocurrencia de las inundaciones se relaciona con las descargas hídricas; en México las lluvias atípicas han causado inundaciones y severos estragos en la infraestructura y en los asentamientos humanos. Los antecedentes sobre las inundaciones asociados a intensas lluvias en 2006, destacan los estragos ocasionados en la infraestructura y a la población de las zonas marginadas. Las fuertes precipitaciones en la cabecera de las cuencas y la deforestación provocan avenidas e intensos escurrimientos con una gran cantidad de material de arrastre y sedimentos en suspensión, lo cual ha provocado modificaciones en los cauces, reduciendo su área hidráulica (CAEM, 2007). En la Zona Metropolitana de Toluca (ZMT), Estado de México, el crecimiento de la población es un factor de presión que incrementa la demanda de tierras para el uso habitacional, desbordando el crecimiento hacia las áreas no aptas para la urbanización (DOF, 1993). El Valle de Toluca se extiende en una planicie con pendientes suaves de 2 a 5\%; al sur de la ciudad capital se encuentran las laderas del Volcán Nevado de Toluca, constituidas por terrenos arenosos y deforestados, y al producirse los escurrimientos, el agua acarrea materiales que azolvan los cauces de los ríos y el sistema de drenaje. En 2006 se presentaron inundaciones que alcanzaron niveles de 40 y 60 centímetros de altura al oriente y sur de la ZMT, ocasionando daños físicos en las viviendas cercanas, pérdidas económicas y en general impacto en las actividades cotidianas. En este contexto se ubica la trascendencia de evaluar la exposición biofísica de los asentamientos humanos a los riesgos por inundación.

\section{ANTECEDENTES}

Uno de los aspectos que agudizan la exposición al riesgo, es la expansión desordenada de las ciudades, la población de menos recursos ocupa las peores tierras, zonas de laderas inestables o zonas de inundación. Como consecuencia de las condiciones caóticas se prevé un incremento en los niveles de riesgo y por lo tanto en el potencial de ocurrencia de desastres (Mansilla, 2002, p. 1); los gobiernos coinciden en la necesidad de generar estrategias de actuación, prevención y mitigación en el marco de la planificación y el desarrollo urbano. Los factores de riesgo en el ámbito urbano son múltiples y dependen del contexto en el que tienen lugar. Los rasgos del riesgo urbano, son los siguientes: a) La velocidad del cambio, b) la degradación ambiental urbana, c) la vulnerabilidad del ámbito urbano, y c) la aparición de nuevos riesgos (Park, 1999). Las experiencias resaltan la zonificación de las áreas de riesgo en una 
unidad geográfica y su jerarquización, de acuerdo con criterios biofísicos, socioeconómicos e institucionales (Tabla 1).

Tabla 1. Síntesis de las aportaciones metodológicas de los estudios de caso

\begin{tabular}{|c|c|c|c|}
\hline Caso/Autor/año & Enfoque & Objetivo & Factores \\
\hline CIDIAT (1984) & $\begin{array}{l}\text { Diagnóstico } \\
\text { físico } \\
\text { conservacionista } \\
\text { en cuencas } \\
\text { hidrográficas. }\end{array}$ & $\begin{array}{l}\text { Determinar el estado } \\
\text { de conservación o } \\
\text { deterioro en una } \\
\text { cuenca hidrográfica. }\end{array}$ & $\begin{array}{l}\text { Erosión. } \\
\text { Clima, relieve } \\
\text { vegetación. }\end{array}$ \\
\hline Cabrera (1987) & $\begin{array}{l}\text { Impacto } \\
\text { ambiental. }\end{array}$ & $\begin{array}{l}\text { Identificar en la cuenca } \\
\text { superior del río Chixoy, } \\
\text { Guatemala, áreas } \\
\text { vulnerables. }\end{array}$ & $\begin{array}{l}\text { Biofísicos (causas } \\
\text { naturales). } \\
\text { Sitios de alto potencial } \\
\text { de aporte de } \\
\text { sedimentos. }\end{array}$ \\
\hline $\begin{array}{l}\text { CENAPRED } \\
(2006)\end{array}$ & Espacial. & $\begin{array}{l}\text { Mostrar la } \\
\text { manifestación } \\
\text { espacial de la } \\
\text { estimación del riesgo } \\
\text { por inundaciones. }\end{array}$ & $\begin{array}{l}\text { Datos de precipitación } \\
\text { de más de } 20 \text { años. } \\
\text { Mapas de peligro por } \\
\text { precipitaciones. }\end{array}$ \\
\hline $\begin{array}{l}\text { Garnica } \\
\text { Alcántara } \\
(2004)\end{array}$ & $\begin{array}{l}\text { Hidrológico. } \\
\text { Evaluación del } \\
\text { riesgo. }\end{array}$ & $\begin{array}{llr}\text { Analizar } & & \text { la } \\
\text { vulnerabilidad a los } \\
\text { riesgos } & & \text { por } \\
\text { inundación en el } \\
\text { curso bajo del río } \\
\text { Tecolutla, Veracruz. }\end{array}$ & $\begin{array}{l}\text { Criterios morfológicos y } \\
\text { estadísticos, técnicas } \\
\text { de percepción remota. }\end{array}$ \\
\hline $\begin{array}{ll}\text { Talamantes } & \text { y } \\
\text { Gurría (2010) } & \end{array}$ & Vulnerabilidad. & $\begin{array}{l}\text { Analizar la incidencia } \\
\text { de avenidas } \\
\text { catastróficas en la } \\
\text { cuenca del río Blanco, } \\
\text { Veracruz. }\end{array}$ & $\begin{array}{l}\text { Zonas de inicio de } \\
\text { avenidas catastróficas. } \\
\text { Condiciones de } \\
\text { degradación en cada } \\
\text { una de las subcuencas. }\end{array}$ \\
\hline
\end{tabular}

En México se han desarrollado diversos estudios relacionados con el tema de investigación, los cuales se resumen a continuación.

Garnica y Alcántara (2004) realizaron un estudio hidrológico en el curso bajo del río Tecolutla, Veracruz; a través del análisis de vulnerabilidad, determinaron el riesgo de la región y establecieron que aproximadamente el $30 \%$ de la población es susceptible a algún tipo de riesgo, en particular las áreas susceptibles a inundarse. Para realizar el trabajo utilizaron criterios morfológicos y estadísticos, y técnicas de percepción remota. La metodología que siguieron puede servir de guía para estudiar áreas con la misma problemática. 
En este sentido el CENAPRED (2006) elaboró mapas de peligro por precipitaciones a partir de datos de las estaciones meteorológicas registradas por el Servicio Meteorológico Nacional, en las cuales se tienen registros de más de 20 años, sin embargo estos registros solo se han analizado en un periodo de retorno de cinco años, utilizándose para el diseño de obras de protección, tales como drenajes, drenes y alcantarillas.

Uno de los estudios más representativos y con mayor relación con el tema de investigación es el de Talamantes y Gurría (2010), en él se hace un análisis de incidencia de efectos presentes en la cuenca del río Blanco, Veracruz, determinan las zonas de inicio de avenidas catastróficas, y las condiciones de degradación en cada una de las subcuencas presentes. Utilizaron la jerarquización y la superposición de diferentes parámetros. En el estudio se delimitaron las microcuencas de la cuenca del río Blanco, y se tomaron en cuenta las condiciones climáticas de la región, el uso del suelo, la pendiente del terreno de los ríos, entre otros, que tienen un efecto importante sobre el tiempo de respuesta de la cuenca hidrográfica.

\section{METODOLOGÍA}

El alcance de la investigación está definido por el objetivo y el diseño operacional de las variables e indicadores. Partimos de la relación estrecha que existe entre la amenaza de un fenómeno natural, la vulnerabilidad y el riesgo que se produciría en un sitio, comunidad, región o país (Figura 1).

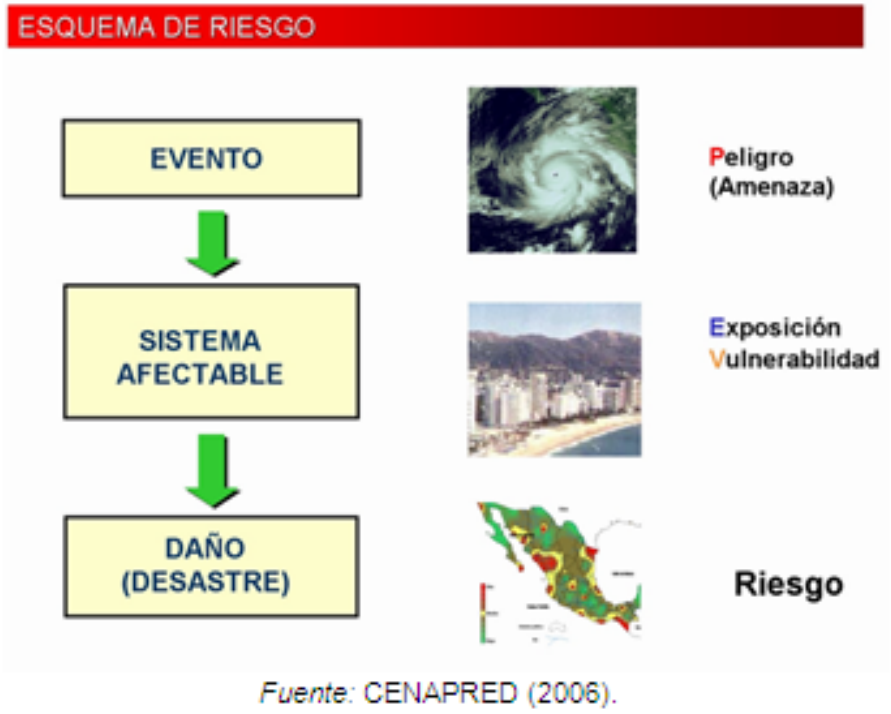

Figura 1. Esquema de riesgo.

La amenaza se comprende como la ocurrencia potencial de un fenómeno natural, en un intervalo de tiempo y un área geográfica específica. La vulnerabilidad, susceptibilidad o propensión de los sistemas expuestos a ser afectados o dañados por el efecto de un fenómeno perturbador, es decir el grado de pérdidas esperadas 
(CENAPRED, 2006). El riesgo es el grado esperado de pérdida (de elementos vulnerables) debido a fenómenos naturales particulares (amenaza), entonces Riesgo = Amenaza + Vulnerabilidad.

El procedimiento metodológico comprende tres niveles de análisis, en la determinación de estos niveles se consideran los objetivos de la investigación (Figura 2).

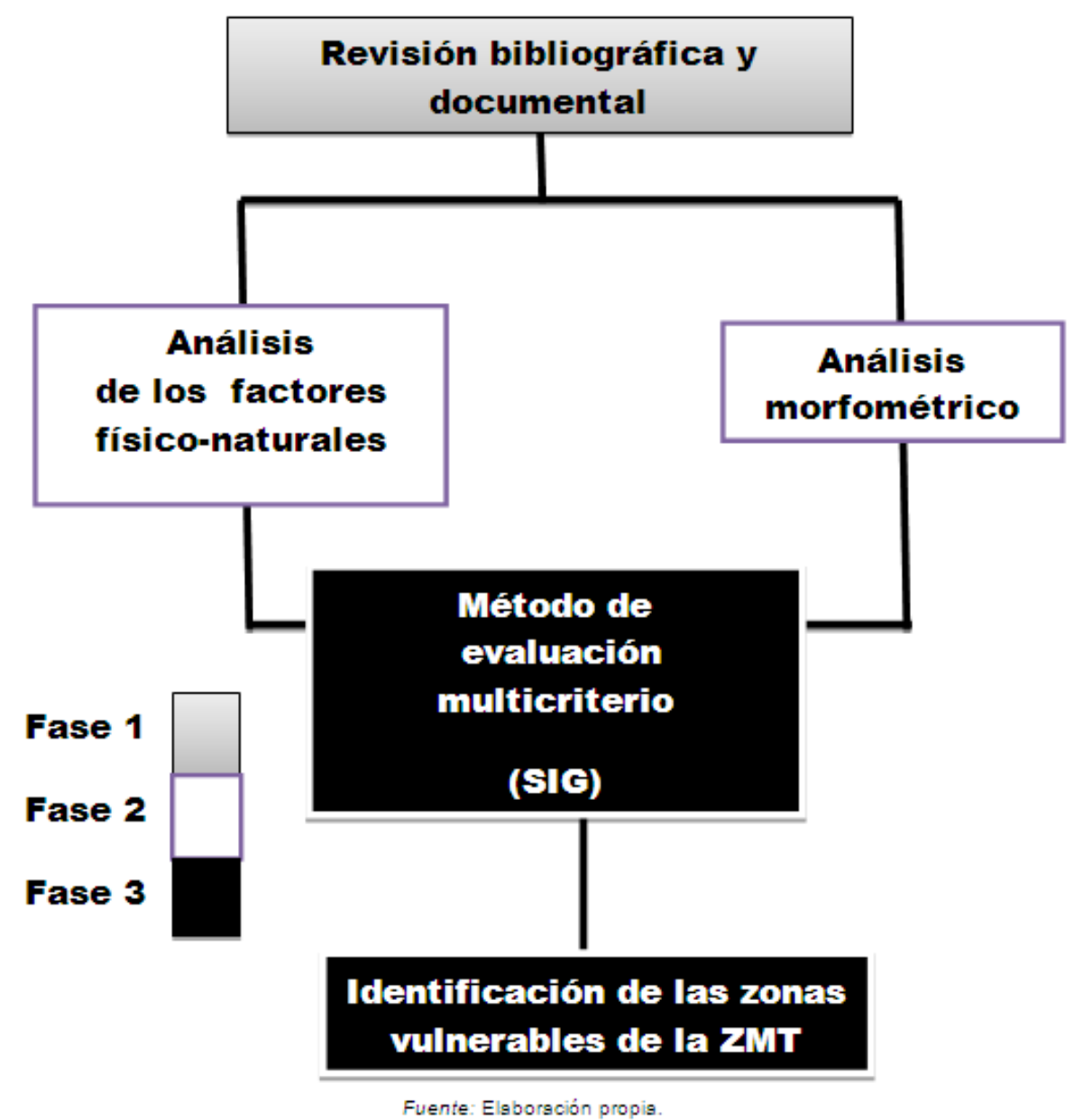

Figura 2. Etapas de análisis.

\section{Equipo y materiales}

Las fuentes de datos para la investigación, incluyen la información proporcionada por diversos organismos que dependen de los gobiernos federal, estatal y municipal, entre ellos datos censales, imágenes satelitales, ortofotos, cartografía temática, reportes y estudios específicos, cartas temáticas sobre climas, edafología, uso de suelo y vegetación, corrientes superficiales y subterráneas en formato digital a escala 1: 20.000, 1: 50.000 y 1: 250 y los censos de población de los años 1990, 2000 y 2010.

Para fundamentar los aspectos teóricos y empíricos de los riesgos por inundación en los asentamientos humanos se realizó la identificación, revisión y análisis de literatura que comprendió el campo del conocimiento del urbanismo y el campo de conocimiento 
de los riesgos naturales, y por medio de los Sistemas de Información Geográfica ArcGIS 9.2 se realizó la selección de las variables biofísicas y se diseñaron las bases de datos alfanumérica y cartográfica, para la manipulación, extracción y análisis de la información. Para poder realizar la cartografía del análisis y zonificación de la vulnerabilidad biofísica, se utilizó el método EMC, que es un conjunto de técnicas orientadas a asistir en los procesos de toma de decisiones y el cual se encuentra desarrollado ampliamente dentro del SIG. En el apartado siguiente se describen los procesos que involucra dicho método.

\section{Método de Evaluación Multicriterio}

El Método de Evaluación Multicriterio (EMC) utiliza los principios del proceso de análisis jerárquico desarrollado por Saaty (1980); está diseñado para resolver problemas complejos de criterios múltiples. Constituye un conjunto de técnicas de gran utilidad para investigar el territorio y coadyuvar a la gestión sustentable de los recursos naturales. Se trata de evaluar un conjunto de alternativas (unidades espaciales de observación) a la luz de múltiples criterios y prioridades en conflicto. Desde el punto de vista geográfico, las alternativas suelen ser un conjunto de porciones del territorio que son evaluadas con base en sus características ambientales, sociales o económicas (Barredo, 1996, p. 47). Un parte fundamental de este método es la matriz de decisión la (aij) la cual "[...] expresa las cualidades (valor numérico o simbólico) de la alternativa o unidad de observación i con respecto a los n atributos considerados" (Barba-Romero y Pomerol, 1997, p. 27) (Tabla 2). El conjunto de elección se refiere a las alternativas o entidades geográficas diferentes, caracterizadas por una serie de atributos que, cuando se les añade un mínimo de información relativa a las preferencias del decisor, se consideran criterios. Si los criterios corresponden a evaluaciones numéricas, se les denomina cuantitativos y cuando no presentan una unidad canónica de medida, se les conoce como cualitativos.

Tabla 2. La matriz de decisión

\begin{tabular}{|c|c|c|c|c|c|c|c|}
\hline \multicolumn{8}{|c|}{ Atributos o criterios } \\
\hline A & & $\mathrm{C}_{1}$ & $\mathrm{C}_{2}$ & $\ldots$ & Cj & $\ldots$ & $\mathrm{Cn}$ \\
\hline I & $\mathrm{A}_{1}$ & $\mathrm{~A}_{11}$ & $\mathrm{~A}_{12}$ & & $a_{1 j}$ & $\ldots$ & $a_{1 n}$ \\
\hline $\mathrm{t}$ & $\mathrm{A}_{2}$ & $\mathrm{~A}_{21}$ & $\mathrm{~A}_{22}$ & & $a_{2 j}$ & $\ldots$ & $a_{2 n}$ \\
\hline e & $"$ & $"$ & $"$ & & $"$ & $"$ & “ \\
\hline$r$ & $"$ & $"$ & $"$ & & “ & “ & “ \\
\hline $\mathrm{n}$ & $"$ & “ & “ & & $"$ & “ & “ \\
\hline al & " & " & $"$ & & " & $"$ & " \\
\hline $\mathrm{t}$ & $"$ & $"$ & “ & & $"$ & $"$ & “ \\
\hline i & $"$ & $"$ & $"$ & $\ldots$ & $"$ & $"$ & “ \\
\hline $\mathrm{v}$ & $"$ & $"$ & $"$ & $\ldots$ & “ & $"$ & “ \\
\hline $\mathbf{a}$ & $"$ & $"$ & $"$ & $\ldots$ & $"$ & $"$ & " \\
\hline s & $\mathrm{A}_{\mathrm{j}}$ & $a_{1 j}$ & $a_{2 j}$ & $\ldots$ & $\mathrm{a}_{1 \mathrm{j}}$ & $\ldots$ & ain \\
\hline
\end{tabular}


Dado que los valores de dicha matriz pueden ser estimados por el decisor en función de diversas unidades y escalas de medida, Saaty (1980) justificaba la elección de la siguiente escala de juicios (Tabla 3):

Tabla 3. Escala de juicios de valor propuesta por Saaty (1980)

\begin{tabular}{|c|c|c|}
\hline Escala numérica & Escala verbal & Explicación \\
\hline 1 & Igual importancia. & $\begin{array}{l}\text { Los dos elementos contribuyen } \\
\text { igualmente a la propiedad o criterio. }\end{array}$ \\
\hline 3 & $\begin{array}{l}\text { Moderadamente más importante un } \\
\text { elemento que otro. }\end{array}$ & $\begin{array}{l}\text { El juicio y la experiencia previa } \\
\text { favorecen a un elemento frente al otro. }\end{array}$ \\
\hline 5 & $\begin{array}{l}\text { Fuertemente más importante un } \\
\text { elemento que otro. }\end{array}$ & $\begin{array}{l}\text { El juicio y la experiencia previa } \\
\text { favorecen fuertemente a un elemento } \\
\text { frente al otro. }\end{array}$ \\
\hline 7 & $\begin{array}{l}\text { Mucho más fuerte la importancia de } \\
\text { un elemento que otro }\end{array}$ & $\begin{array}{l}\text { Un elemento domina fuertemente. Su } \\
\text { dominación está probada en práctica. }\end{array}$ \\
\hline 9 & $\begin{array}{l}\text { Importancia extrema de un } \\
\text { elemento frente al otro. }\end{array}$ & $\begin{array}{l}\text { Un elemento domina al otro con el } \\
\text { mayor orden de magnitud posible. }\end{array}$ \\
\hline
\end{tabular}

De acuerdo con Barba-Romero y Pomerol (1997), existen diversas razones que justifican la utilización de la escala propuesta. Ellas son: el amplio abanico de posibilidades sin superar el número de valores que la mente humana puede contemplar simultáneamente y el uso de valoraciones enteras con incrementos (Franco-Maass, 2009).

\section{Morfometría}

Una de las herramientas más importantes en el análisis hídrico, es la morfometría. Esta, permite establecer parámetros de evaluación del comportamiento morfodinámico e hidrológico del sistema, así como analizar y comprender los elementos geométricos básicos del mismo (Méndez y Marcucci, 2006).

Para la determinación de los parámetros morfométricos se usó el software ArGIS 9.2 y la extensión DetermHidro (Valtierra, 2007). Como base topográfica se usaron las curvas de nivel con cotas de $10 \mathrm{~m}$, obtenidas de las cartas topográficas digitales que proporciona el Instituto Nacional de Estadística Geografía e Informática (INEGI). En función de estas capas se realizó un Modelo Digital de Elevación (MDE), como base para modelar los flujos de agua y determinar la red de drenaje de la Zona Metropolitana de Toluca.

\section{ANÁLISIS DE LOS FACTORES FÍSICO-NATURALES QUE PROVOCAN LAS INUNDACIONES EN LA ZMT}

El subsistema físico-natural o medio físico está constituido por el territorio propiamente dicho y sus recursos. Su relación con los asentamientos humanos de la ZMT, se han establecido a partir del análisis del medio ambiente natural a partir de los aspectos como la 
edafología, la hidrología, el uso de suelo y vegetación y la población, como determinantes de esta relación. El objetivo de analizar el subsistema natural o medio físico es conocer cómo se utiliza el territorio y sus recursos naturales, incluyendo las degradaciones y amenazas que actúan sobre el mismo. Otro aspecto a considerar, es determinar la fragilidad o vulnerabilidad del territorio.

\section{Ubicación de la zona de estudio}

La unidad espacial en la que se realizó la investigación está conformada por el Valle de Toluca localizado en el centro-oeste del Estado de México. Se constituye en el altiplano más meridional de la cuenca del río Lerma hasta la Presa José Antonio Alzate. La ZMT se ubica en la porción central del territorio estatal, entre los $19^{\circ} 02^{\prime}$ y $19^{\circ} 32^{\prime}$ LN y $99^{\circ} 19^{\prime}$ y $99^{\circ} 56^{\prime}$ LO (Figura 3). Esta porción territorial comprende los 14 municipios que conforman la Zona Metropolitana ${ }^{3}$ de Toluca y cubre una superficie de 1943 km2 albergando a 1'846.116 habitantes (INEGI, 2010).

Figura 3. Zona Metropolitana de Toluca en el contexto del Estado de México y de la República Mexicana

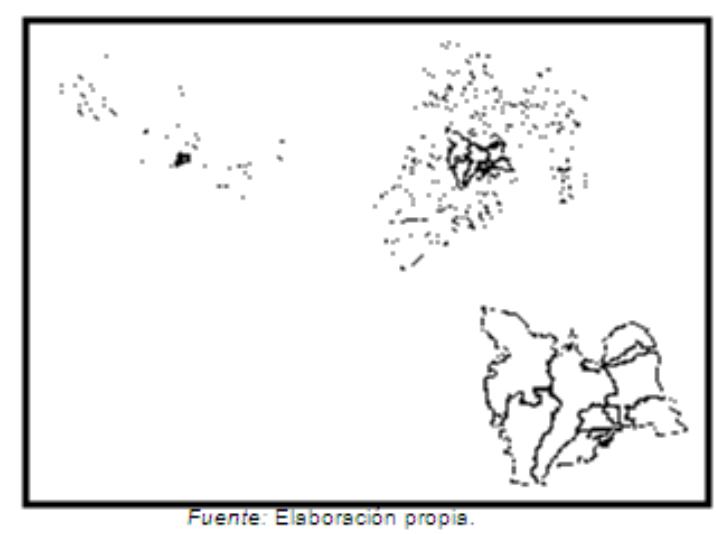

Clasificación de la vulnerabilidad edafológica

La relación suelo-roca es importante para la formación de suelos, para el sostén de construcciones humanas y para la obtención del recurso agua, procesos que guardan estrecha relación con los asentamientos humanos.

En la ZMT se localizan 10 grupos edáficos de los 38 establecidos en el mapa mundial de suelos de la FAO/UNESCO (1988), donde el Feozem es el suelo con mayor distribución en la zona cubriendo un $42 \%$ de la misma. Una vez realizada la clasificación edafológica se aplicó el método EMC al factor mencionado y se obtuvo la vulnerabilidad edafológica; los resultados se observan en el siguiente Mapa. 


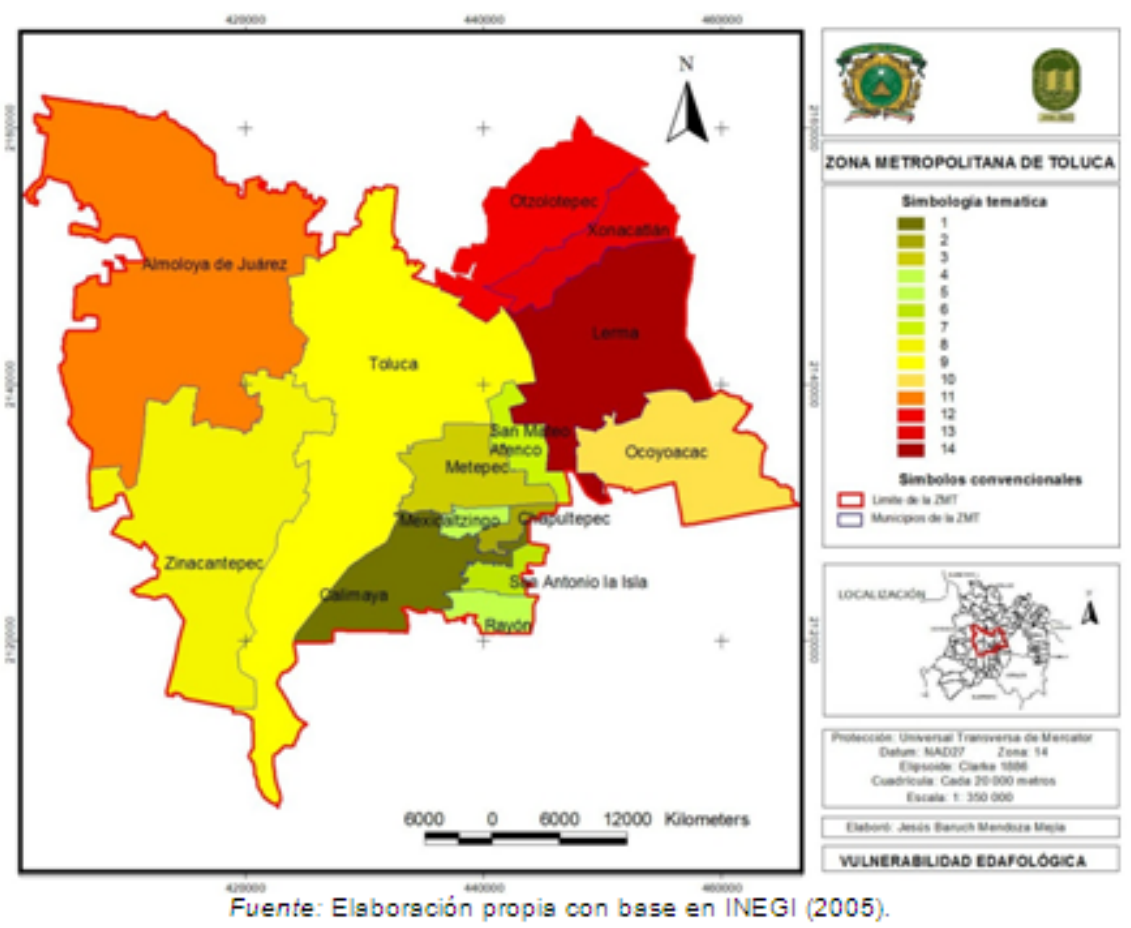

Mapa 1. Clasificación de la vulnerabilidad edafológica.

En la Tabla siguiente se observa que la mayor parte de la ZMT presenta una vulnerabilidad baja con un $41 \%$ del total del área de estudio correspondiente a una superficie de $840,82 \mathrm{~km}^{2}$, mientras que los municipios de Lerma, Chapultepec, Calimaya y Almoloya de Juárez presentan una vulnerabilidad alta con un $39,80 \%$ del total de la ZMT que corresponden a $807,27 \mathrm{~km}^{2}$.

Tabla 4. Valores de la clasificación de la vulnerabilidad edafológica y área afectada

\begin{tabular}{lccc}
\hline \multicolumn{1}{c}{ Municipio } & $\begin{array}{c}\text { Área } \\
\left(\mathbf{k m}^{2}\right)\end{array}$ & $\begin{array}{c}\text { Valor asignado } \\
\text { por el EMC }\end{array}$ & $\begin{array}{c}\text { Clasificación de la } \\
\text { vulnerabilidad }\end{array}$ \\
\hline Xonacatlán & 65,34 & 1 & BAJA \\
Toluca & 453,60 & 2 & BAJA \\
San Mateo Atenco & 30,34 & 3 & BAJA \\
San Antonio la Isla & 18,37 & 4 & BAJA \\
Rayón & 23,14 & 5 & BAJA \\
Otzolotepec & 115,17 & 6 & BAJA \\
Ocoyoacac & 134,86 & 7 & BAJA \\
Zinacantepec & 301,19 & 8 & MEDIA \\
Mexicaltzingo & 11,92 & 9 & MEDIA \\
Metepec & 67,15 & 10 & MEDIA \\
Lerma & 210,79 & 11 & ALTA \\
Chapultepec & 12,77 & 12 & ALTA \\
Calimaya & 100,16 & 13 & ALTA \\
Almoloya de Juárez & 483,55 & 14 & ALTA \\
\hline
\end{tabular}




\section{Clasificación de la vulnerabilidad del uso de suelo y vegetación}

La cobertura vegetal y los usos del suelo más actualizados para el Estado de México, son los determinados en el Inventario Nacional Forestal (INF) 2000-2001, elaborado por el Instituto de Geografía de la Universidad Nacional Autónoma de México (UNAM), para la SEMARNAP y del cual se obtiene la tabla que muestra el tipo de vegetación y los usos del suelo, mismos que han sido utilizados en el presente estudio. La cobertura de uso de suelo y vegetación incluye las aplicaciones (tipo de ocupación y uso existente), ya sea agropecuarias-forestales o los espacios urbanos.

Aplicando el método EMC a las coberturas de uso de suelo y vegetación antes mencionadas, se obtuvieron los siguientes resultados:

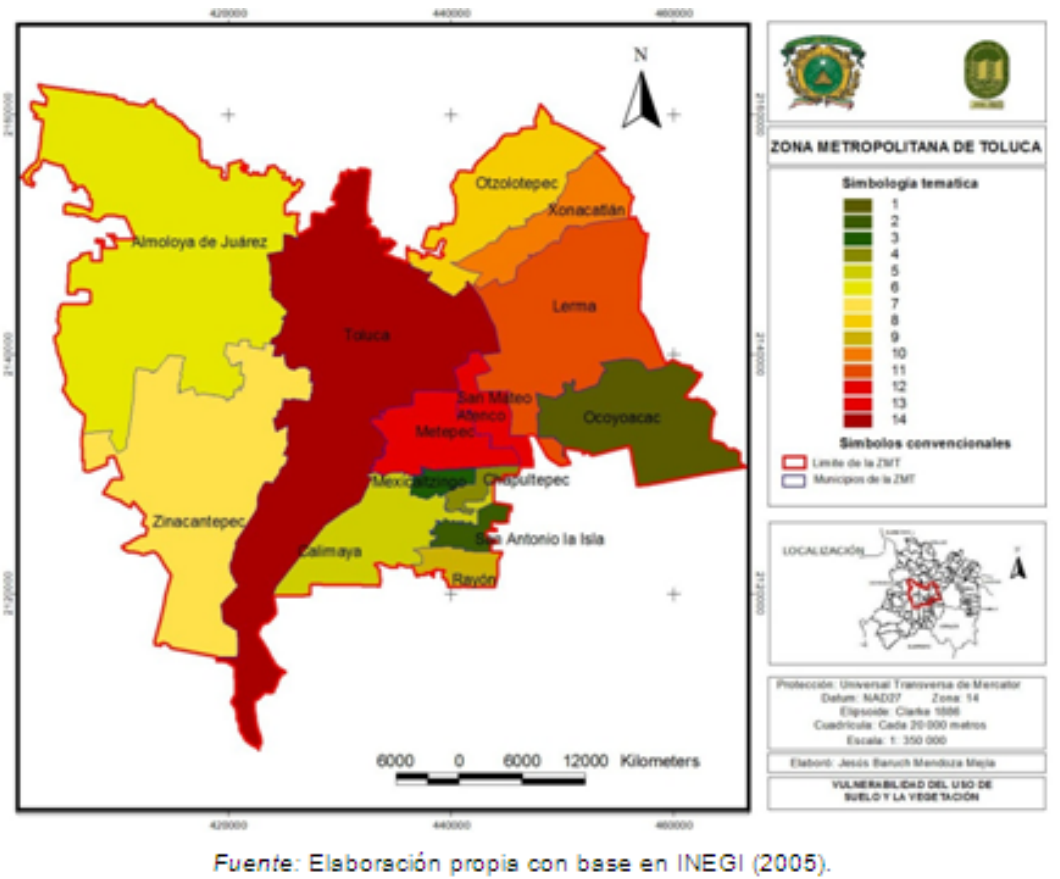

Mapa 2. Clasificación de la vulnerabilidad del uso de suelo y la vegetación.

En la Tabla siguiente se observa que la vulnerabilidad alta del uso de suelo y la vegetación predomina en la ZMT con un $56,30 \%$ con una superficie de 1.142,01 km2, mientras que la vulnerabilidad media es únicamente de 3,9\% del total de la zona de estudio. Los municipios que presentaron una vulnerabilidad alta son: Zinacantepec, Xonacatlán, Toluca, San Mateo Atenco, San Antonio la Isla, Rayón, Otzolotepec y Ocoyoacac. 
Tabla 5. Valores de la clasificación del uso de suelo y vegetación y área afectada

\begin{tabular}{lccc}
\hline Municipio & Área $\left(\mathbf{k m}^{2}\right)$ & $\begin{array}{c}\text { Valor asignado por } \\
\text { el EMC }\end{array}$ & $\begin{array}{c}\text { Clasificación de la } \\
\text { vulnerabilidad }\end{array}$ \\
\hline Zinacantepec & 301,19 & 14 & ALTA \\
Xonacatlán & 65,34 & 13 & ALTA \\
Toluca & 453,60 & 12 & ALTA \\
San Mateo Atenco & 30,34 & 11 & ALTA \\
San Antonio la Isla & 18,37 & 10 & ALTA \\
Rayón & 23,14 & 9 & ALTA \\
Otzolotepec & 115,17 & 8 & ALTA \\
Ocoyoacac & 134,86 & 7 & ALTA \\
Mexicaltzingo & 11,92 & 6 & MEDIA \\
Metepec & 67,15 & 5 & MEDIA \\
Lerma & 210,79 & 4 & BAJA \\
Chapultepec & 12,77 & 3 & BAJA \\
Calimaya & 100,16 & 2 & BAJA \\
Almoloya de Juárez & 483,55 & 1 & BAJA \\
Funte-Elgborgcín propis & & &
\end{tabular}

Clasificación de la vulnerabilidad hidrológica

El Valle de Toluca es el lugar de nacimiento de la cuenca hidrológica del río Lerma-Santiago, una de las más grandes e importantes de México. Siguiendo el sentido del cauce del río Lerma, el valle se termina al norte, en el llamado estrechamiento de Perales, 9 kilómetros sobre el curso del río Lerma después de la Presa José Antonio Alzate. Lugar donde se localizan las fallas transversales que definen los límites de las subcuencas del Valle de Toluca e Ixtlahuaca-Atlacomulco, Estado de México.

En los últimos 50 años los acuíferos de esta zona se han visto abatidos por la extracción constante de los volúmenes de agua para el Distrito Federal que es la capital del país, provocando entre otros factores que los niveles de perforación de pozos sean cada vez más profundos. Aplicando el método EMC al factor hidrológico se obtuvieron los siguientes resultados: 


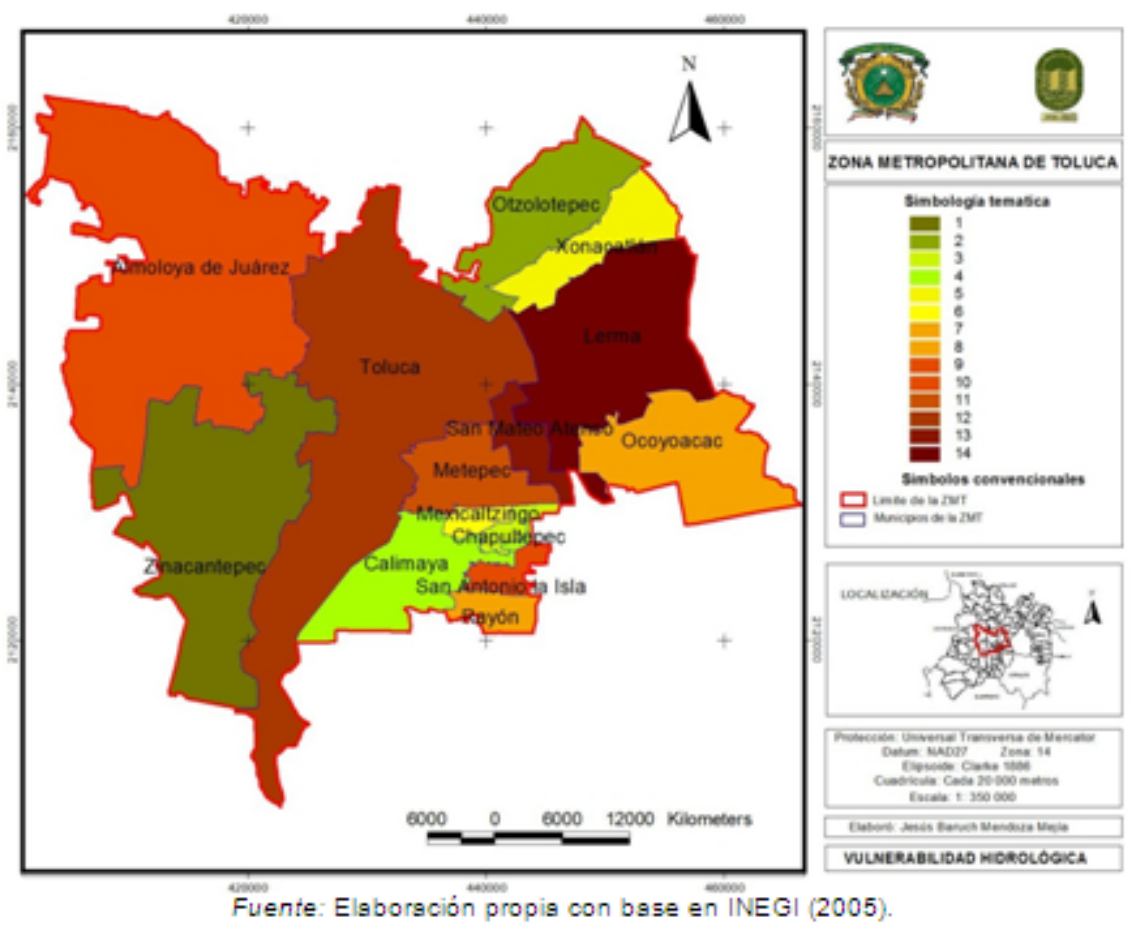

Mapa 3. Clasificación de la vulnerabilidad hidrológica.

En la Tabla siguiente se observa que la vulnerabilidad hidrológica alta cubre el $70,10 \%$ de la ZMT, la cual representa una superficie de $1.421,80 \mathrm{~km}^{2}$; los municipios pertenecientes a esta clasificación son: Rayón, Ocoyoacac, San Antonio la Isla, Almoloya de Juárez, Metepec, Toluca, San Mateo Atenco y Lerma. Con una vulnerabilidad media se encuentran los municipios de Mexicaltzingo y Xonacatlán con el $26,09 \%$ del total de la zona de estudio.

Tabla 6. Valores de la clasificación de la vulnerabilidad hidrológica y área afectada

\begin{tabular}{lccc}
\hline Municipio & Area $\left(\mathbf{k m}^{2}\right)$ & $\begin{array}{c}\text { Valor asignado por } \\
\text { el EMC }\end{array}$ & $\begin{array}{c}\text { Clasificación de la } \\
\text { vulnerabilidad }\end{array}$ \\
\hline Zinacantepec & 301,19 & 1 & BAJA \\
Otzolotepec & 115,17 & 2 & BAJA \\
Calimaya & 100,16 & 3 & BAJA \\
Chapultepec & 12,77 & 4 & BAJA \\
Mexicaltzingo & 11,92 & 5 & MEDIA \\
Xonacatlán & 65,34 & 6 & MEDIA \\
Rayón & 23,14 & 7 & ALTA \\
Ocoyoacac & 134,86 & 8 & ALTA \\
San Antonio la Isla & 18,37 & 9 & ALTA \\
Almoloya de Juárez & 483,55 & 10 & ALTA \\
Metepec & 67,15 & 11 & ALTA \\
Toluca & 453,60 & 12 & ALTA \\
San Mateo Atenco & 30,34 & 13 & ALTA \\
Lerma & 210,79 & 14 & ALTA \\
Fuente: Elsborsción propis. & & &
\end{tabular}

\section{Parámetros morfométricos de la ZMT}

A continuación se describen los resultados de los parámetros principales así como los valores de estos para la cuenca. 


\section{a) Curva hipsométrica}

Refleja el equilibrio de la cuenca y la identifica en sus etapas de A) juventud, B) madurez y C) vejez (Senciales y Ferre, 1992). El Gráfico resultante se muestra a continuación:

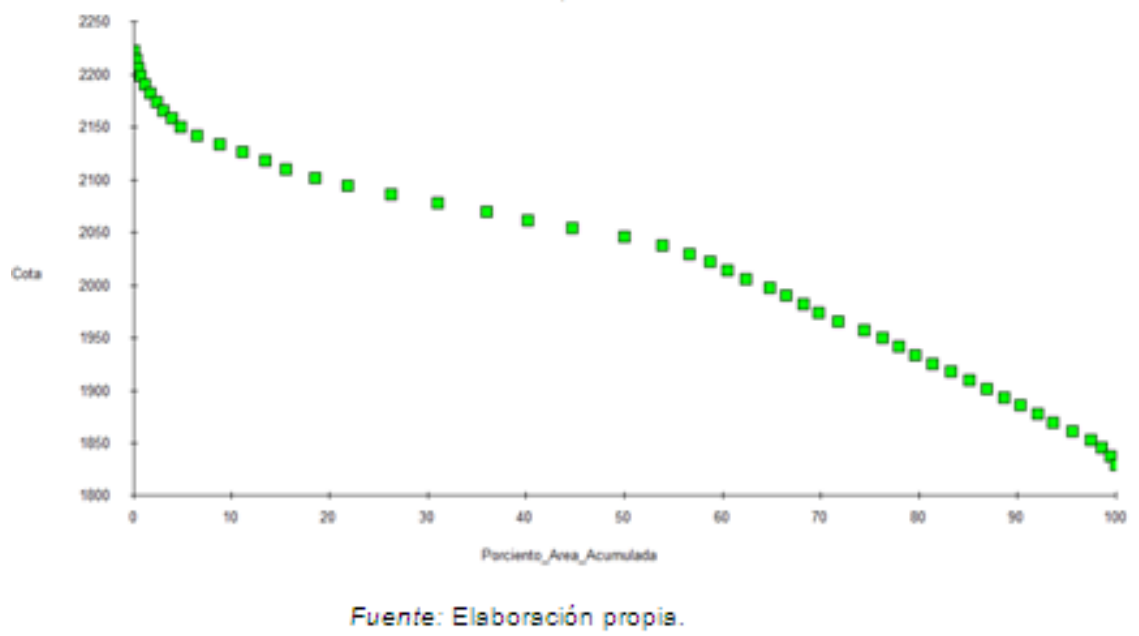

Gráfico 1. Curva hipsométrica de cuenca de la ZMT.

De acuerdo a la forma obtenida del Gráfico, se puede observar que el tipo de cuenca indica una clara tendencia hacia la etapa $C$ o de vejez, en donde se puede interpretar la cuenca con fuertes procesos de erosión debido al tránsito de las escorrentías superficiales.

b) Pendiente media del cauce

Indica la pendiente media del río o cauce principal y su potencial para erosionar, íntimamente relacionado con la edad de la cuenca (Campos, 1987). Para su determinación se hace uso de los siguientes valores:

Tabla 7. Indicadores de pendiente de la cuenca de la ZMT

\begin{tabular}{ccc}
\hline Concepto & Valor & Unidades \\
\hline Pendiente media & 20 & $\%$ \\
Elevación mínima & 2600,00 & $\mathrm{msnm}$ \\
Elevación máxima & 4392,65 & $\mathrm{msnm}$ \\
Elevación media & 2804,93 & $\mathrm{msnm}$ \\
Longitud del cauce principal & 16,08 & $\mathrm{~km}$ \\
\hline Fuente: Elsborgción propis. & &
\end{tabular}

Distribución y clasificación de la vulnerabilidad de la población en la ZMT

La ciudad de Toluca inicia su configuración como Zona Metropolitana a partir de la década de los 60 , derivado de la 
industrialización del corredor Toluca-Lerma y que a su vez significó cambios importantes de actividades productivas y sociales en estructura socioeconómica del Valle de Toluca, motivando la integración de municipios al conjunto metropolitano (COESPO, 2010).

El volumen poblacional de la ZMT ha aumentado 6,3 veces en promedio entre 1950 y 2010; hoy cuenta con 1'846.116 habitantes asemejándose a un crecimiento lineal constante, al menos por población absoluta. Los municipios que más veces han aumentado su población en el periodo referido son: Metepec con 12,5, San Mateo Atenco $(7,9)$ y Toluca $(7,1)$. Aplicando el método EMC a la distribución poblacional antes mencionada se obtuvieron los siguientes resultados:

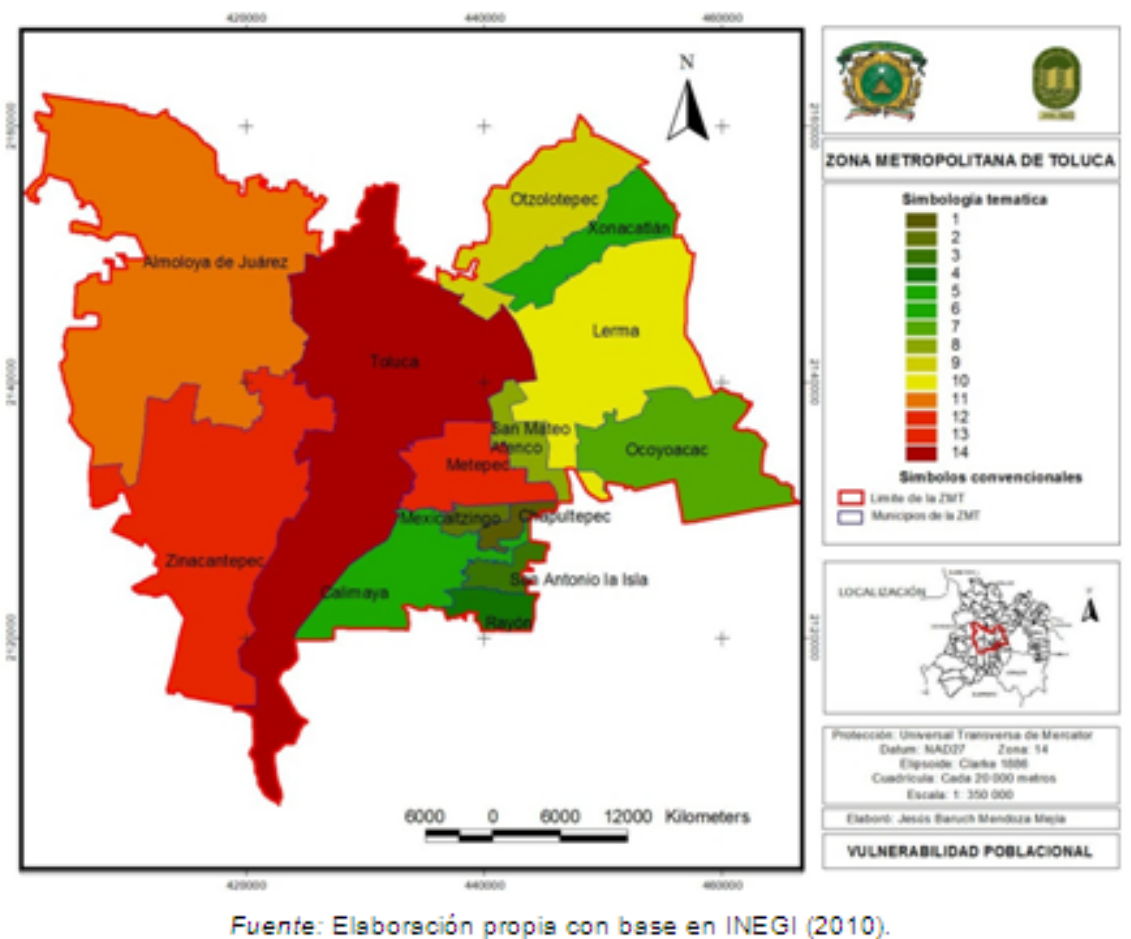

Mapa 4. Clasificación de la vulnerabilidad poblacional.

En la Tabla siguiente se observa que la vulnerabilidad poblacional alta predomina en la ZMT con un $64,3 \%$ con $1305,49 \mathrm{~km}^{2}$, mientras que la vulnerabilidad media es de únicamente de $10,39 \%$ del total de la zona de estudio. Los municipios que presentaron una vulnerabilidad alta son: Almoloya de Juárez, Metepec, Toluca y Zinacantepec. 
Tabla 8. Valores de la clasificación de la vulnerabilidad poblacional

\begin{tabular}{lccc}
\hline Municipio & Area $\left(\mathbf{k m}^{2}\right)$ & $\begin{array}{c}\text { Valor asignado por el } \\
\text { EMC }\end{array}$ & $\begin{array}{c}\text { Clasificación de la } \\
\text { vulnerabilidad }\end{array}$ \\
\hline Zinacantepec & 301,19 & 12 & ALTA \\
Xonacatlán & 65,34 & 6 & BAJA \\
Toluca & 453,60 & 14 & ALTA \\
San Mateo Atenco & 30,34 & 8 & BAJA \\
San Antonio la Isla & 18,37 & 3 & BAJA \\
Rayón & 23,14 & 4 & BAJA \\
Otzolotepec & 115,17 & 9 & BAJA \\
Ocoyoacac & 134,86 & 7 & BAJA \\
Mexicaltzingo & 11,92 & 2 & BAJA \\
Metepec & 67,15 & 13 & ALTA \\
Lerma & 210,79 & 10 & MEDIA \\
Chapultepec & 12,77 & 1 & BAJA \\
Calimaya & 100,16 & 5 & BAJA \\
Almoloya de Juárez & 483,55 & 11 & ALTA \\
Fuente: Elgborsción propis. & & &
\end{tabular}

\section{Integración de indicadores de vulnerabilidad para determinar el} riesgo biofísico en la ZMT

La integración de los indicadores analizados anteriormente se realizó mediante la aplicación del EMC a resultados obtenidos de los mismos, esto permitió obtener el Mapa siguiente en el que se refleja el riesgo biofísico de la ZMT.
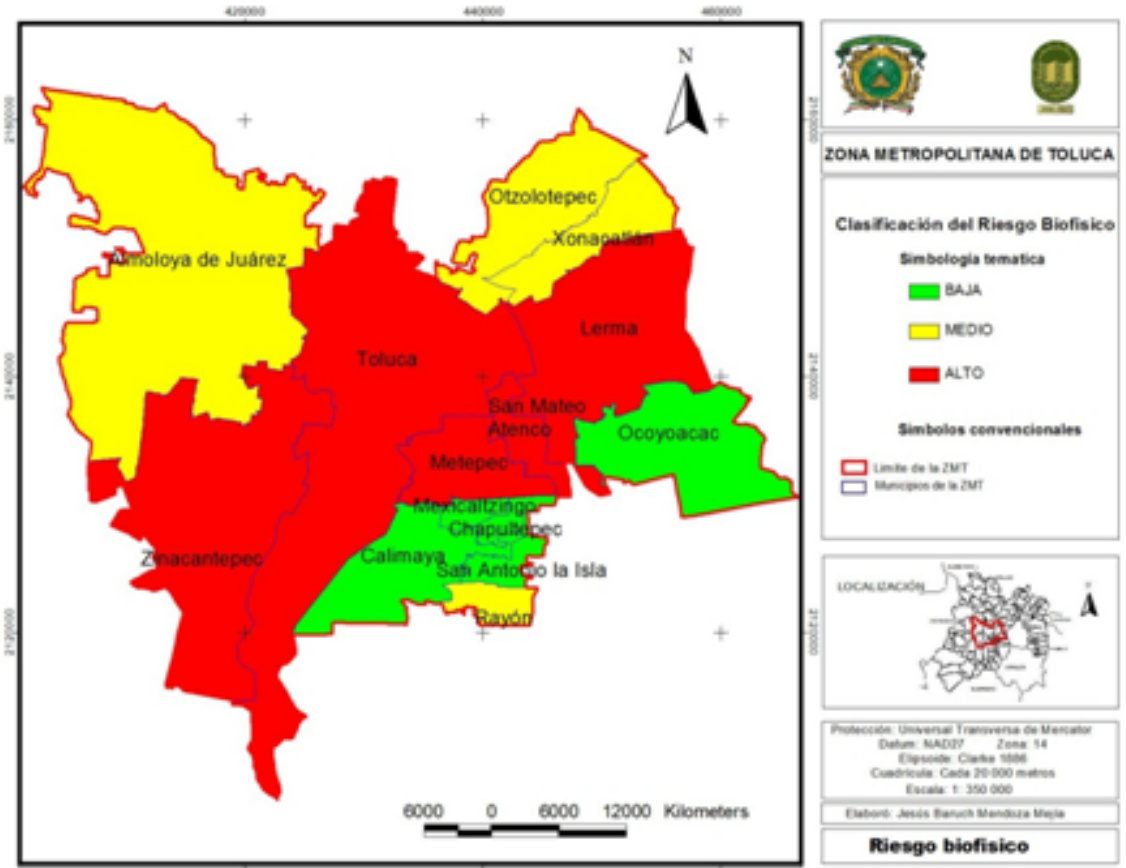

Fuente: Elsborsción propis con bsse en INEGI (2010)

Mapa 5. Clasificación de riesgo biofísico en la ZMT.

En la Tabla siguiente se observa que en términos generales la mitad de la ZMT presenta un riesgo biofísico alto con un valor de $52,41 \%$, este porcentaje comprende los municipios de Lerma, Toluca, San Mateo Atenco y Metepec y cubre una superficie de 1032,73 km². En 
cuanto a la clasificación del riesgo biofísico medio, éste representa una superficie considerable de la ZMT ya que la superficie que cubre es de $687,20 \mathrm{~km}^{2}$ y representa el $33,88 \%$ del total de la zona.

Por último, la clasificación que presenta menores valores tanto en porcentaje como en superficie cubierta fue el riesgo biofísico bajo, ya que únicamente cubre el $13,71 \%$ del total de la ZMT con una superficie de $13,71 \mathrm{~km}^{2}$. Los valores antes mencionados son el resultado de clasificaciones de alta vulnerabilidad que presentaron los factores del uso de suelo y vegetación, la población y la hidrología, a excepción del factor edafológico que presentó un $39,80 \%$ del total de la ZMT, sin embargo este valor no está muy alejado de los otros factores clasificados como altos, lo cual significa que tiene una tendencia a convertirse en valor alto.

Tabla 9. Clasificación del riesgo biofísico en la ZMT

\begin{tabular}{lccc}
\hline Municipio & Área $\left(\mathbf{k m}^{2}\right)$ & $\begin{array}{c}\text { Valor asignado por } \\
\text { el EMC }\end{array}$ & $\begin{array}{c}\text { Clasificación de la } \\
\text { vulnerabilidad }\end{array}$ \\
\hline Almoloya de Juárez & 483,55 & 6 & MEDIA \\
Calimaya & 100,16 & 1 & BAJA \\
Chapultepec & 12,77 & 2 & BAJA \\
Lerma & 210,79 & 11 & ALTA \\
Metepec & 67,15 & 13 & ALTA \\
Mexicaltzingo & 11,92 & 4 & BAJA \\
Ocoyoacac & 134,86 & 5 & BAJA \\
Otzolotepec & 115,17 & 8 & MEDIA \\
Rayón & 23,14 & 7 & MEDIA \\
San Antonio la Isla & 18,37 & 3 & BAJA \\
San Mateo Atenco & 30,34 & 10 & ALTA \\
Toluca & 453,60 & 14 & ALTA \\
Xonacatlán & 65,34 & 9 & MEDIA \\
Zinacantepec & 301,19 & 12 & ALTA \\
Fuente:Elaborsción propis. & & &
\end{tabular}

\section{CONCLUSIONES}

La cuenca hidrográfica, por ser la unidad física en la cual tienen lugar todos los procesos naturales, es asimismo la unidad natural y lógica para el desarrollo agrícola, ambiental y socioeconómico. Con el crecimiento demográfico y el aumento de las necesidades de urbanización, industrialización y producción de alimentos, los efectos de la actividad antropogénica ya no se limitan solo a zonas pequeñas ni a una comunidad en particular. Las fuertes precipitaciones en la parte alta de esta, así como la deforestación de la misma, han contribuido a que al presentarse las avenidas, el escurrimiento venga acompañado de una gran cantidad de material de arrastre y sedimento en suspensión. El peligro potencial a inundaciones y avenidas, combinado con la alta vulnerabilidad, de algunas comunidades tanto física como socioeconómicamente condicionan el nivel de riesgo al que están expuestas.

De acuerdo a lo anterior, se puede afirmar que la ZMT se encuentra dentro de una cuenca de tipo $C$ (de acuerdo a la curva hipsométrica resultante de la morfometría), geológicamente representa una 
cuenca con valles extensos y cumbres escarpadas, por lo que ha sido sometida a un proceso intenso de erosión. Lo anterior está estrechamente relacionado con el uso del tipo de vegetación, la cual ha disminuido en un $10 \%$ en los últimos 10 años. Esta ausencia de cubierta vegetal suele incrementar las velocidades con que se mueve el agua en la cuenca, reduce la cantidad de agua infiltrada en el suelo y la interceptada por la vegetación.

Otro factor importante es la textura del suelo, la cual también influye en la velocidad de infiltración del agua. En este caso en la ZMT contiene texturas arcillosas, las cuales originan más encharcamientos.

Con lo mencionado anteriormente, podría decirse que la combinación de los factores mencionados contribuyen a provocar zonas inundables, aunado a otros factores como las precipitaciones extraordinarias. Se espera que los resultados obtenidos contribuyan en la toma de decisiones de diferentes sectores como protección civil o el desarrollo regional; también pueden ser utilizados en otros aspectos específicos como en el diseño de los programas de reordenamiento territorial con una visión de riesgo, apoyo a los planes de seguridad pública a través de los programas de protección civil o en adecuación de los planes de desarrollo urbano mediante la incorporación de información sobre riesgos hidrometeorológicos.

\section{REFERENCIAS}

- Barba-Romero, S. y Pomerol, J.-C. (1997). Decisiones Multicriterio. Fundamentos teóricos y utilización práctica. Madrid: Ed. Universidad de Alcalá de Henares.

- Barredo Cano, J.I. (1996). Sistemas de información geográfica y evaluación multicriterio en la ordenación del territorio. Madrid: RAMA. pp. 1-58.

- Cabrera C., 1987. Identificación de áreas críticas con base en criterios biofísicos y análisis básico de la degradación específica y transporte de sedimentos en la cuenca superior del río Chixoy, Guatemala. Tesis Mag. Sc. Turrialba, CR, CATIE. 174 p.

- CAEM -Comisión del Agua del Estado de México-. (2007). Atlas de inundaciones 14, Cuenca del río Lerma.

- Campos Aranda, D.F. (1987). Procesos del ciclo hidrológico. San Luis Potosí: Editorial Universitaria Potosina. pp. 2-15.

- CENAPRED -Centro Nacional de Prevención de Desastres-. (2006). Elaboración de mapas de riesgo por inundaciones y avenidas súbitas en zonas rurales, con arrastre de sedimentos. México: Coordinación de Investigación, Área de Riesgos Hidrometeorológicos.

- CIDIAT (Centro Interamericano de Desarrollo Integral de Aguas y Tierras, VE) / MARNR (Ministerio del Ambiente y de los Recursos Naturales Renovables, VE), 1984. Diagnóstico físico conservacionista en cuencas hidrográficas. Mérida, VE, CIDIAT. 63 $\mathrm{p}$.

- COESPO -Consejo Estatal de Población-. (2010). Centro de Documentación del Consejo Estatal de Población. 
- DOF -Diario Oficial de la Federación-. (1993). Ley General de Asentamientos Humanos. Publicada el 21 de julio de 1993.

- FAO/UNESCO(1988).Manual de Campo para la ordenación de cuencas hidrográficas. Estudio y planificación de Cuencas hidrológicas.

- Franco-Maass, S. (2009). Aplicación de las técnicas de evaluación multicriterio para la caracterización agroecológica del territorio. En Reyes, G. (Comp.), Acercamientos conceptuales y metodológicos para el estudio de la realidad agropecuaria y rural de México (pp. 1351). Toluca: ICAR, UAEM.

- Garnica, R.J. y Alcántara, I. (2004). Riesgos por inundación asociados a eventos de precipitación extraordinaria en el curso bajo del río Tecolutla, Veracruz. Investigaciones Geográficas, Boletín del Instituto de Geografía, UNAM, 55, 23-45. Disponible en: http://www.revistas.unam.mx/index.php/rig/article/view/30109

- INEGI -Instituto Nacional de Estadística Geografía e Informática-. (2005). Cartografía temática escala 1: 250 000. Serie III.

- . (2010). Conteo de Población y Vivienda.

- Mansilla, E. (2002). La ciudad: El nuevo escenario del riesgo. Oficina de Planificación del Área Metropolitana de San Salvador (OPAMSS). Mayo, 2002. San Salvador, El Salvador.

- Méndez, W. y Marcucci, E. (2006). Análisis morfométrico de la microcuenca Quebrada Curucutí, Estado de Vargas-Venezuela. Revista Geográfica Venezolana, 47(1).

- Saaty, T.L. (1980). Multicriteria Decision Making: The Analytic Hierarchy Process. New York: McGraw-Hill. (2a impresión 1990, RSW Pub. Pittsburgh).

- SEDESOL/CONAPO/INEGI -Secretaría de Desarrollo Social / Consejo Nacional de Población / Instituto Nacional de Estadística, Geografía e Informática- (2005). Delimitación de las zonas metropolitanas de México. Primera edición. México.

- Senciales, J.M. y Ferre, E. 1992. Análisis morfométrico de la cuenca del río Benamargosa (provincia de Málaga). En López Bermúdez, F.; Conesa, C. y Romero, M.A. (Eds.): Estudios de Geomorfología en España. Actas de la II Reunión Nacional de Geomorfología, Murcia, S.E.G., pp. 365-375.

- Park, R. E. (1999). La ciudad y otros ensayos de ecología urbana. Barcelona: Ediciones del Sebal.

- Talamantes, P. y Gurría, A. (2010). Análisis de incidencia de efectos presentes en la cuenca del río Blanco, Veracruz. México: Universidad Autónoma de Querétaro. pp. 1-57.

- Valtierra, J.G. (2007). Desarrollo de una herramienta computacional para el diagnóstico hidrológico de cuencas. Tesis de Maestría. Maestría en Gestión Integrada de Cuencas. Universidad Autónoma de Querétaro.

1. Estudiante del Doctorado en Urbanismo de la Facultad de Planeación Urbana y Regional, UAEM. baruchmm@yahoo.com.mx

2. Profesor Investigador de la Facultad de Planeación Urbana y Regional y Centro de Investigación en Estudios Avanzados en Planeación Territorial, UAEM. eorozcoh61@hotmail.com

3. Zona metropolitana, conjunto de dos o más municipios donde se localiza una ciudad de 50 mil o más habitantes, cuya área urbana, funciones y actividades rebasan el límite del municipio que 
originalmente la contenía, incorporando como parte de sí misma o de su área de influencia directa a municipios vecinos, predominantemente urbanos, con los que mantiene un alto grado de integración socioeconómica; en esta definición se incluye además a aquellos municipios que por sus características particulares son relevantes para la planeación y política urbanas (SEDESOL/CONAPO/INEGI, 2005).

Para citar este artículo: Mendoza Mejía, J. B. \& Orozco Hernández, M. E. (2014). Análisis de la vulnerabilidad biofísica a los riesgos por inundación en la zona metropolitana de Toluca, México. Revista Luna Azul, 38, 86-104. Recuperado de http://lunazul.ucaldas.edu.co/index.php?option=content\&task=v iew\&id=895 